\title{
Efeito da oclusão temporal na ação de ataque sobre a tomada de decisão defensiva na modalidade de voleibol
}

CDD. 20.ed. 152.3

796.325

http://dx.doi.org/10.1590/1807-55092015000100107

\author{
Fábio Luis BORDINI'/** \\ Marcelo Alves COSTA** \\ Josiane MEDINA-PAPST ${ }^{* *}$ \\ Thiago Viana CAMATA** \\ Inara MARQUES ${ }^{* * *}$
}

*Departamento de

Educação Física,

Centro Universitário

Filadélfia.

* * Universidade

Estadual de Londrina.

\section{Resumo}

0 presente estudo analisou o efeito da oclusão temporal na cortada do voleibol sobre a tomada de decisão defensiva em atletas com diferentes niveis de experiência. Os participantes foram divididos em três grupos: adulto $(G A D ; n=16)$, infanto/mirim $(G I M ; n=16)$ e adulto novato (GNO; $n=16)$. Imagens da finalização de jogadas de ataque realizadas por quatro atletas foram editadas em cinco diferentes momentos: (OT1) a $399 \mathrm{~ms}$ (12 quadros) antes do contato do atacante com a bola; (OT2) a $266 \mathrm{~ms}$ (oito quadros) antes; (OT3) a $133 \mathrm{~ms}$ (quatro quadros) antes; (OT4) no momento do contato atacante/bola e; (OT5) a $133 \mathrm{~ms}$ (quatro quadros) após o contato do atacante com a bola. Ao assistirem os vídeos editados, os participantes deveriam informar o local de aterrissagem da bola seguido da confiança com a qual emitiam suas respostas. Foi mensurada a precisão na predição da trajetória da bola (acerto/erro) e a confiança da resposta (escala Likert 1-5). Quanto à frequência de acertos, o grupo $G A D(\bar{X}=63,67$ $\pm 10,38 \%)$ apresentou maior frequência de acertos que $\operatorname{GIM}(\bar{X}=55,46 \pm 10,17 \%)$ em OT2 $(p=0,001)$. A frequência de acertos de GAD ( $X=79,29 \pm 10,38 \%)$ também foi maior que a de GNO $(\bar{X}=71,87 \pm$ $10,43 \%)$ em OT3 $(p=0,012)$. As condições mostraram-se diferentes entre si (Bonferroni's $p<0,005)$, com a frequência de acertos aumentando de OT1 ( $\bar{X}=36,06 \pm 12,44 \%)$ à OT5 ( $\bar{X}=98,17 \pm 4,81 \%)$. Para confiança, GAD e GIM apresentaram-se mais confiantes que GNO (Bonferroni's $p<0,016$ ) em OT1, OT2, 0T3. Novamente, as condições diferiram entre si (Bonferroni's $p<0,005)$, com os grupos mostrando-se mais confiantes em 0T5. Concluiu-se que, independente da experiência, os grupos se mostraram capazes de predizer a localização de aterrissagem da bola. Contudo, grupos com maior experiência mostraram-se superior quanto à sua capacidade antecipatória.

Palavras-Chave: Tomada de decisão; Antecipação; Padrão de reconhecimento visual; Voleibol.

\section{Introdução}

Por muito tempo, no esporte, acreditou-se que a superioridade individual devia-se a uma espécie de seleção natural, na qual o sucesso dos atletas adviria por um sistema nervoso mais desenvolvi$\mathrm{do}^{1}$. Contudo, nas últimas três décadas, estudiosos no esporte têm fornecido evidências de que, em modalidades esportivas que apresentam restrições temporais, o desempenho habilidoso não se refere apenas à realização precisa da ação motora ${ }^{2}$. Assim, diversas modalidades esportivas foram investigadas, tais com: hóquei de campo ${ }^{1}$, diferentes esportes com raquete $^{3-10}$, futebol ${ }^{11}$, cricket $^{12}$, rúgbi $^{13}$, e voleibol quadra e praia ${ }^{2,14-15}$.

A partir disso, houve uma crescente conscientização no que diz respeito à importância de habilidades cognitivas perceptuais para o desempenho em alto nível ${ }^{16}$. Tais habilidades fazem referência à capacidade de identificar e adquirir informaçôes ambientais a fim de selecionar e executar respostas apropriadas ${ }^{17}$. Desta forma, não só a habilidade motora, mas também a efetiva aquisição e processamento de informações visuais especificas à tarefa apresentariam relevância ${ }^{2}$. 
Devido ao atraso inerente ao processamento da informação no sistema perceptual humano ${ }^{18}$, um atleta é frequentemente solicitado a tomar decisóes baseadas em informaçôes visuais parciais, a fim de realizar respostas motoras apropriadas ${ }^{14}$. A capacidade de predição de indivíduos utilizando-se de fontes parciais e relevantes de informaçáo foi o que PoulTON $^{19}$ se referiu como antecipaçáo perceptiva. Assim, a capacidade antecipatória do atleta desempenha um papel de extrema importância a fim de alcançar patamares elevados em decisóes bem sucedidas ${ }^{20}$.

Nos mais variados esportes destaca-se a ideia de que jogadores experientes se mostram superiores aos seus pares menos experientes quando solicitados a predizer o resultado de uma determinada ação ${ }^{21}$. Contudo, apesar da inclusão de grupos com experiência intermediária em determinados estudos ${ }^{1,6,12,22-23}$, sua relação com indivíduos com maior experiência e com novatos ainda não demonstram-se claras.

No esporte, para Chamberlain e Coelho ${ }^{24}$, um ponto adicional que discrimina indivíduos com diferentes experiências é a confiança com a qual os mesmos tomam suas decisóes. De acordo com JACKSON e MorGAN ${ }^{10}$, avaliar a confiança com a qual os indivíduos emitem suas respostas nos possibilita maior compreensão quanto as suas decisôes. Contudo, vale ressaltar que ainda náo há consenso na literatura de que mais experientes também apresentem uma maior confiança em suas respostas ${ }^{13,23,25}$, náo estando claro se

\section{Método}

\section{Amostra}

Participaram do estudo 48 indivíduos do sexo masculino, divididos igualmente em três grupos: Grupo Adulto (GAD), composto por atletas da categoria Adulto (19 anos ou mais) com média de idade de 27,3 $( \pm 6,8)$ anos e tempo de prática sistemática de 13,1 ( \pm 5,7) anos; Grupo Infanto e Mirim (GIM), composto por atletas das categorias Infanto e Mirim (16-17 anos) com média de idade de 17 ( \pm $0,4)$ anos e tempo de prática sistemática de 2,2 ( \pm 1) anos; e Grupo de Novatos (GNO) composto por universitários (20-25 anos), com média de idade de $22,2( \pm 1,5)$ anos, sem experiência competitiva na modalidade. Como critério de inclusão os participantes do GAD e do GIM deveriam ter participado de alguma competição estadual nos últimos dois anos, e os do GNO não poderiam ter experiência a confiança na tomada de decisão pode ser fator determinante no desempenho. Apesar da confiança com que se tomam as decisóes, a literatura ainda destaca a crucialidade da capacidade de predizer a ação de um oponente, formulando assim uma resposta apropriada pautada em consideraçóes táticas e técnicas, para o desempenho bem sucedido no esporte.

Visto que as habilidades de antecipação e tomada de decisão são presumidamente importantes para os atletas alcançarem mais altos graus de excelência $^{26-27}$. Diferentes abordagens metodológicas são utilizadas para avaliação de tais abordagens (cf. GASPAR et $\mathrm{al}^{28}$ ), sendo uma delas o paradigma da oclusão ${ }^{3}$. Nos estudos que utilizam à oclusão temporal, uma sequência de ação (vídeo) é paralisada em determinado ponto de sua execução na tentativa de identificar períodos de tempo dentro das quais atletas experientes consigam identificar o resultado final desta ação baseado em dicas visuais relevantes.

Deste modo, o paradigma da oclusão temporal ${ }^{3}$ foi utilizado para analisar o efeito do tempo de prática e das oclusóes temporais na precisão das respostas, relacionando-as com a confiança da tomada de decisão em uma ação de defesa no voleibol. Apesar dos estudos já realizados dentro deste paradigma de análise, poucos estudos analisaram o voleibol de qua$\mathrm{dra}^{14}$. Ademais, não tem sido foco de estudo a análise da capacidade antecipatória na tomada de decisão defensiva, em função da experiência dos jogadores.

na modalidade de voleibol. Os pais ou o responsável legal pelos participantes do GIM e os participantes do GAD e GNO foram previamente informados sobre os objetivos da pesquisa e os procedimentos a serem adotados, juntamente com os técnicos das equipes a que os atletas de GAD e GIM pertenciam. Após os esclarecimentos foi solicitado que assinassem o Termo de Consentimento Livre e Esclarecido. Esse estudo foi aprovado pelo Comitê de Ética em Pesquisa da Universidade Local (parecer n. 125/10; CAAE n. 0117.0.268.000-10; folha de rosto n. 333024).

\section{Instrumentos e procedimentos}

Preparação das sequências de ataque

A captação das imagens ocorreu em um ginásio com a quadra, rede, antenas e bolas em acordo com 
as regras oficiais da modalidade ${ }^{29}$. Inicialmente, uma equipe se posicionou em uma metade da quadra seguindo o sistema de ataque 5 x 1 (cf. BizzocCHI ${ }^{30}$, p.159) e na área de saque da outra metade da quadra posicionou-se um experimentador. Foi simulada uma situação de jogo na qual as finalizaçôes foram realizadas pelo jogador que atuava na posição 4 (cf. Chamberlain \& Coelho ${ }^{31}$, p.24), sendo os demais jogadores responsáveis pela movimentação de proteção ao ataque (cf. Chamberlain \& CoE$\mathrm{LHO}^{31}$, p.121). Foi solicitado aos atacantes que não realizassem qualquer tipo de movimento na tentativa de confundir o defensor e, a cada finalização, a

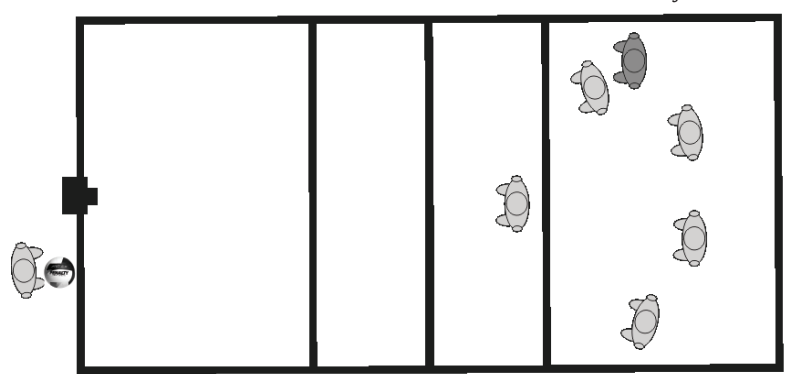

(a) bola foi recolocada em jogo por meio de um saque, sempre considerando o tempo necessário para a reorganização da equipe.

Para captação das imagens, uma câmera (Casio EX-FH20 - $30 \mathrm{~Hz}$ ) foi posicionada a $1,32 \mathrm{~m}$ de altura e centralizada sobre a linha de fundo da quadra contrária à equipe, para simular a visão de um jogador de defesa. Foram filmadas 16 finalizações de quatro diferentes atletas, sendo oito "cortadas" (quatro paralela e quatro diagonal) e oito "largadas" (quatro paralela e quatro diagonal). A posição inicial e as possíveis direçóes das finalizações são representadas esquematicamente na FIGURA 1.

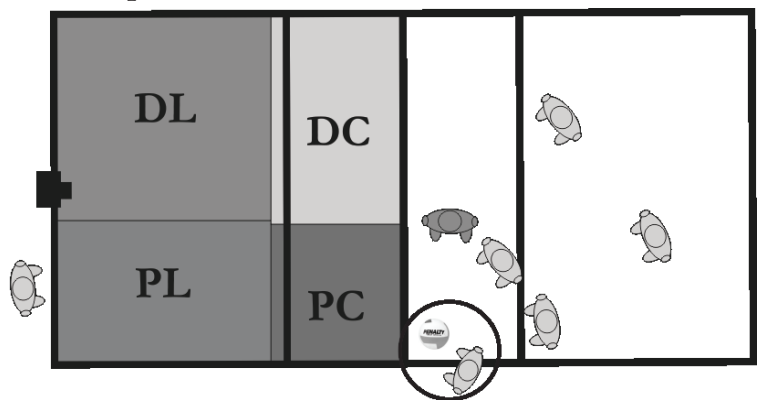

(b)
$\mathrm{PC}=$ paralela curta;

$\mathrm{PL}=$ paralela longa;

$\mathrm{DC}=$ diagonal curta; $\mathrm{DL}=$ diagonal longa.

FIGURA 1 - Representação esquemática: (a) posição inicial dos jogadores e (b) possibilidades de ataque do jogador da posição 4 .

Das finalizaçóes filmadas, 32 foram selecionadas (dois por direção e profundidade por atleta) para inclusão no experimento. Os critérios para inclusão foram: a) $\mathrm{O}$ contato da mão do atacante com a bola deveria ocorrer entre a antena (limite lateral da rede) e uma marcaçáo feita com fita adesiva a 1,2 $\mathrm{m}$ da mesma; b) $\mathrm{O}$ atacante não poderia apresentar qualquer tipo de movimento na tentativa de iludir o defensor (i.e., fintas de corpo).

Depois de selecionados, os vídeos passaram por uma edição ("software" Pinnacle Studio Plus v.11.0), a qual consistiu na interrupção dos mesmos em cinco diferentes momentos ${ }^{14}$. As condições de oclusão foram: (OT1) o vídeo foi interrompido a $399 \mathrm{~ms}$ (12 quadros) antes do contato do atacante com a bola; (OT2) o vídeo foi interrompido a $266 \mathrm{~ms}$ (oito quadros) antes; (OT3) o vídeo foi interrompido a $133 \mathrm{~ms}$ (quatro quadros) antes; (OT4) o vídeo foi interrompido no momento do contato entre a máo do atacante e a bola e; (OT5) o vídeo foi interrompido a $133 \mathrm{~ms}$ (quatro quadros) após o contado do atacante com a bola.

A partir da edição inicial das imagens, foram elaboradas quatro sequências constituídas de 80 vídeos cada, as quais foram divididas em dois blocos de 40, totalizando 16 para cada tipo de oclusão temporal e 20 para cada local de aterrissagem da bola. Partindo das quatro sequências iniciais foram criadas outras quatro, invertendo a ordem de apresentação dos vídeos ("crossover"), totalizando oito sequências, sendo que, cada sequência foi apresentada à apenas seis participantes (dois por grupo). Foi respeitado um intervalo de cinco segundos entre as tentativas e de 60 segundos entre o primeiro e segundo bloco. Estes procedimentos foram adotados a fim de minimizar o efeito do aprendizado e da fadiga por parte dos participantes.

\section{Procedimentos de coleta de dados}

Cada participante foi encaminhado a uma sala (15 m x $10 \mathrm{~m} \times 4 \mathrm{~m}$ ) onde foi representada a metade de uma quadra de voleibol com uma tela de projeção (10 m x 3,2 m) localizada no local da rede. $\mathrm{O}$ participante foi posicionado centralizado a 7,5 m da tela, sendo esta distância designada em razão do jogador inicialmente ocupar esta posição (posição 6) no sistema defensivo com centro recuado (cf. Bizzocchi ${ }^{30}$, p.187). Um projetor de imagens da marca Epson (EMP-S5) foi posicionado a uma altura de 2,5 m estando a $12 \mathrm{~m}$ da tela e, conectado a ele, um computador portátil da marca Toshiba (Satéllite A355-F6935) responsável pela apresentação dos vídeos. A representação esquemática do local de coleta é demonstrada na FIGURA 2. 

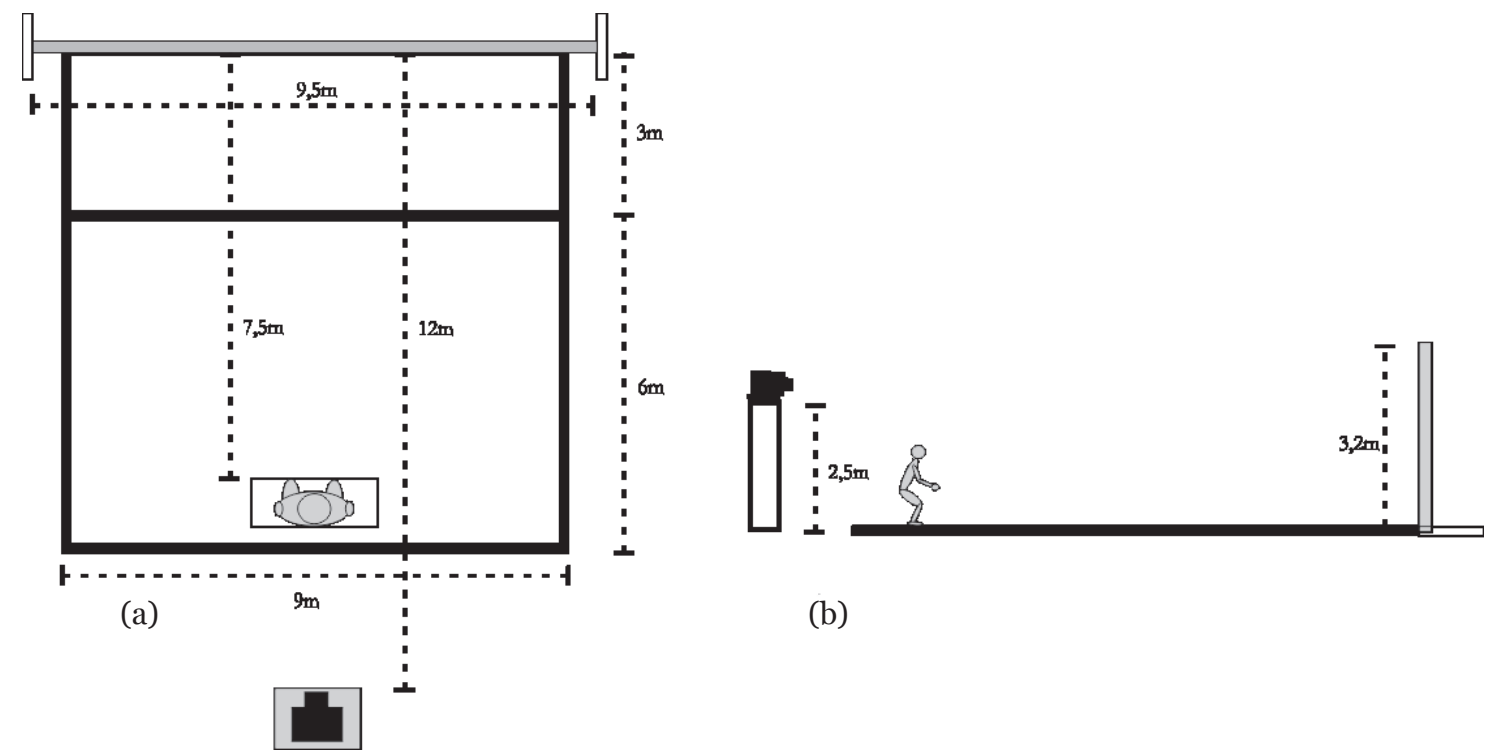

(b)

FIGURA 2 - Representação esquemática do local de coleta: a) vista superior; e b) vista lateral.

No início de cada coleta foi informada ao participante a posição ocupada pela câmera no momento da filmagem, com os participantes devendo considerar as imagens como uma quadra em cenário real. $O$ pesquisador forneceu as instruçôes aos participantes, permitindo também que fossem realizados questionamentos para eliminar qualquer tipo de dúvida. Foi solicitado ao participante que permanecesse na posiçâo de expectativa para defesa a cada imagem projetada, a qual, segundo BOJIKIAN e BoJIKIAN ${ }^{31}$, favorece os deslocamentos para frente ou em diagonal, sendo estes os de maior ocorrência em lances de defesa. Cada participante realizou 10 tentativas para familiarização da tarefa (duas por condição de oclusão), as quais também foram utilizadas para sanar quaisquer dúvidas dos participantes, e, após esse procedimento, foi iniciada a coleta de dados.

A tarefa foi predizer, por meio do deslocamento de seu corpo, o local de aterrissagem da bola no

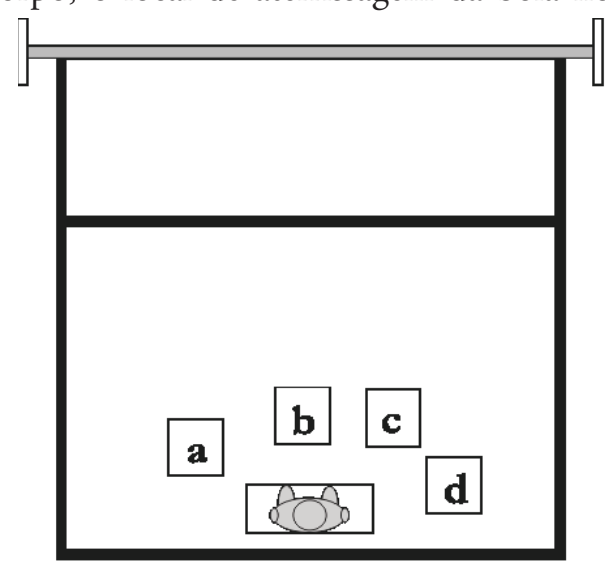

(a) momento da interrupção do vídeo. O participante deveria deslocar-se: a) para a esquerda se identificasse a finalização como uma cortada longa para diagonal; b) para frente e para a esquerda se a ação identificada fosse uma largada curta para a diagonal; c) para frente e para a direita se identificasse que a ação terminaria em uma largada curta para a paralela e, por fim; d) para a direita se identificasse a finalizaçáo como uma cortada longa na paralela. O movimento do corpo como resposta foi utilizado seguindo a proposta de Williams et al. ${ }^{11}$ que examinaram a importância do uso das respostas baseada no movimento para verificar a antecipação no esporte. A distância entre a posiçáo inicial do participante e o local para onde deveria deslocar-se a fim de identificar a localização de aterrissagem da bola foi de aproximadamente $1 \mathrm{~m}$. A representação esquemática tanto das possibilidades de respostas juntamente com as possibilidades de ataque é representada na FIGURA 3.

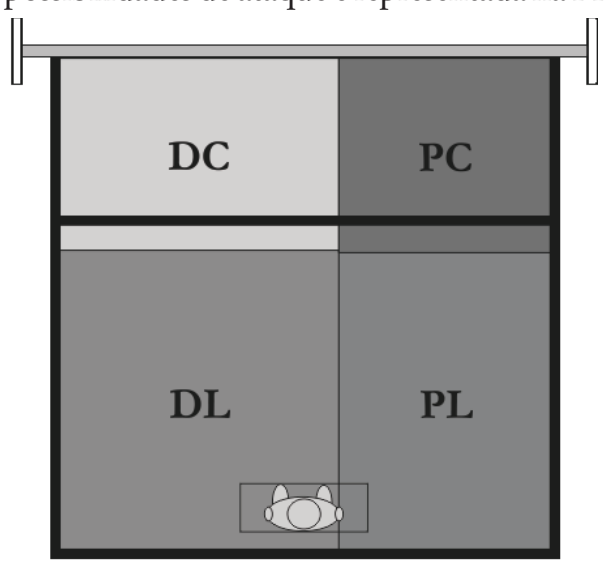

(b)

FIGURA 3 - Representação esquemática: a) possibilidades de respostas e; b) possibilidades de ataques. 
Imediatamente após deslocar-se para o local selecionado, o participante era questionado em relaçáo à sua confiança de resposta. Para analisar a confiança foi utilizada uma escala de Likert, com as seguintes categorias: 1) nada confiante; 2) pouco confiante; 3) confiante; 4) muito confiante e; 5) totalmente confiante. Após a resposta, o participante deveria retomar para a sua posição inicial para a continuidade das tentativas. As respostas dos participantes, tanto da prediçáo quanto da confiança, foram anotadas em uma folha-resposta pelo próprio pesquisador. Foi designado para toda resposta correta o escore " 1 " e para os erros " 0 ". O número de respostas corretas para cada uma das cinco condiçôes de oclusão foi determinado para cada participante.

\section{Resultados}

Os resultados serão apresentados quanto à frequência de prediçóes corretas das respostas de cada um dos grupos de participantes com base na probabilidade de chance de acerto. A probabilidade de chance se refere à possibilidade com que os participantes tinham de acertar as respostas, seja com o máximo de certeza ou mesmo ao acaso.

\section{Análise estatística}

Foi conduzida uma análise estatística descritiva identificando a frequência de acertos (predição) e os valores da mediana e intervalo interquartílico (confiança). Para a análise estatística inferencial utilizou-se o teste KruskalWallis com o "post hoc" de U Mann-Whitney seguido pelo ajuste de Bonferroni para verificar a diferença entre os grupos em cada uma das condiçôes. Para verificar as diferenças entre as condiçôes de oclusão espacial utilizou-se a ANOVA de Friedman com o "post hoc" de Wilcoxon, também seguido pelo ajuste de Bonferroni. Foi utilizado o pacote estatístico SPSS 19.0, com significância estabelecida em $5 \%(p<0,05)$ para os testes principais e o ajuste para os testes "post hoc", sendo calculado o "Effect Size" (ES) para cada uma das comparaçóes.

Posteriormente, serão apresentados os resultados do nível de confiança dessas respostas. A frequência (média e desvio padrão) das prediçôes corretas (\%) de cada grupo, em função das condiçóes de oclusão, e a localização das diferenças estatísticas entre grupos e condiçôes ao longo das tentativas está expressa na FIGURA 4.

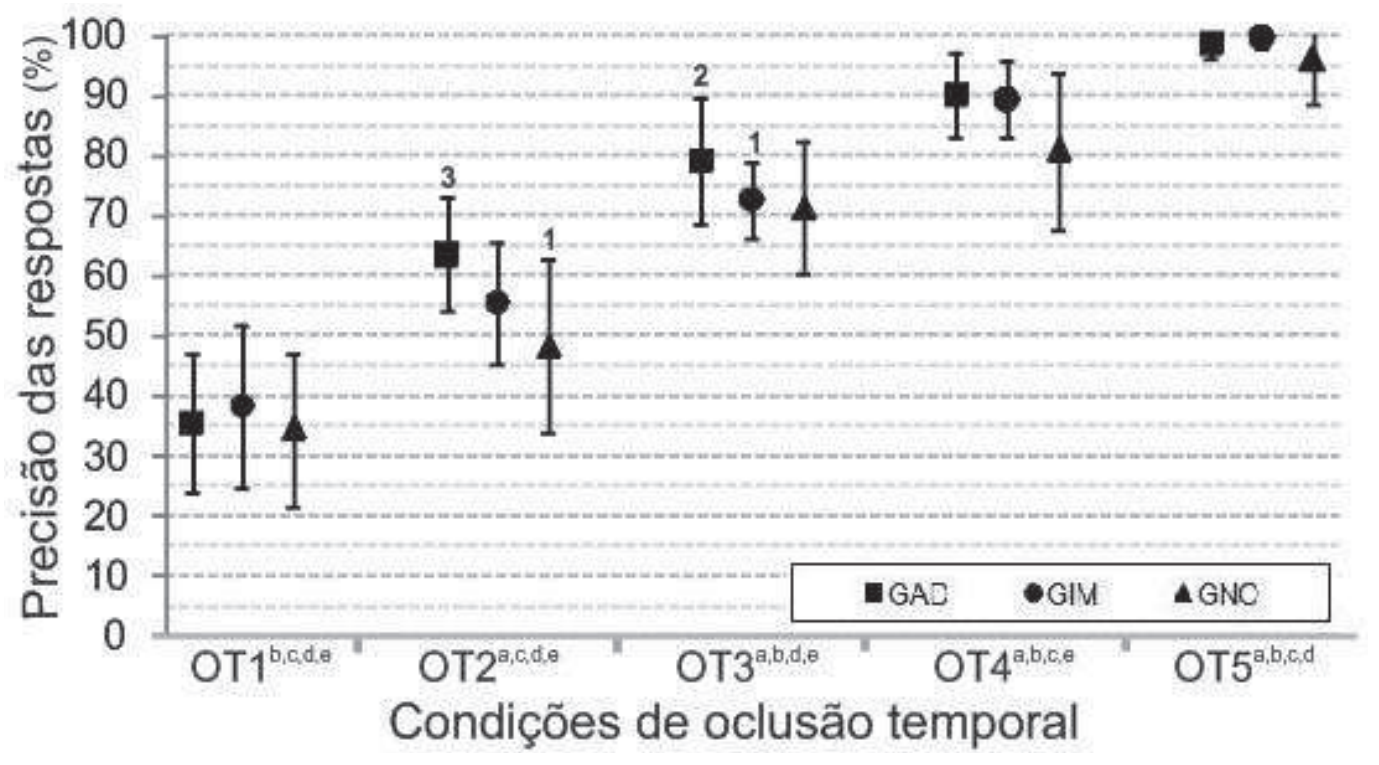

Diferença significativa em comparação aos grupos 1GAD, 2GIM e 3GNO; e às condições aOT1, bOT2, cOT3, dOT4 e eOT5.

FIGURA 4 - Precisão de resposta (média e desvio padrão) dos grupos, em função das condições de oclusão temporal analisadas. 
Os resultados mostraram que, em todas as condições, independente da experiência dos participantes, a média da frequência de acerto dos grupos esteve acima da probabilidade de chance, estabelecido aqui em $25 \%$. Estes resultados indicam que os participantes, independentemente de sua experiência na modalidade, conseguiram, de algum modo extrair informações relevantes para a predição da localização final da bola mesmo a $399 \mathrm{~ms}$ antes do contato entre a mão do atacante e a bola.

$\mathrm{Na}$ análise inferencial para a variável frequência de acertos, o efeito principal foi encontrado para o fator grupo nas condiçóes OT2 $\left(H_{(2)}=12,353 ; p=\right.$ $0,002 ; E S=0,26)$, OT3 $\left(H_{(2)}=7,482 ; p=0,024\right.$; $E S=0,15)$ e OT4 $\left(H_{(2)}=6,311 ; p=0,043 ; E S=\right.$ $0,13)$ e para o fator oclusão $\left(\chi_{(4)}^{2}=179,386\right.$; $p<$ 0,$001 ; E S=0,89$ ).

$\mathrm{Na}$ análise do efeito para o fator grupo, a análise de "post hoc" foi utilizada com a correção de Bonferroni a fim de diminuir o erro tipo I (n. de comparaçôes) indicando a presença de diferenças estatísticas entre $\operatorname{GAD}(\bar{X}=79,30 \pm 10,39)$ e $\mathrm{GNO}(\bar{X}=71,48$ $\pm 10,94)$ em OT2 $(U=41,500 ; p=0,001 ; E S=$ $0,67)$ e entre $\operatorname{GAD}(\bar{X}=63,67 \pm 9,47)$ e $\operatorname{GIM}(\bar{X}=$ $55,47 \pm 10,17)$ em OT3 $(U=63,000 ; p=0,012 ; E S$ $=0,51)$ nas quais, em ambas as comparaçóes, GAD se mostrou com uma frequência de acerto superior a dos demais grupos. O efeito encontrado entre os grupos para OT4 não foi sensível após a correção utilizada (Bonferroni's $p>0,016$ ).

$\mathrm{Na}$ análise do efeito para o fator oclusão, a análise de "post hoc" foi seguido novamente pela correção de Bonferroni. A frequência de acerto da OT1 $(\bar{X}=$ $36,07 \pm 12,47)$ apresentou-se menor que OT2 $(\bar{X}=$ $55,86 \pm 12,98)(Z=-5,187 ; p<0,001 ; E S=0,80)$, OT3 $(\bar{X}=74,48 \pm 9,89)(Z=-6,042 ; p<0,001 ; E S$ $=1,00)$, de OT4 $(\bar{X}=86,85 \pm 10,99)(Z=-6,049 ; p$ $<0,001 ; E S=1,00)$ e de OT5 $(\bar{X}=-98,18 \pm 4,82)(Z$ $=-6,049 ; p<0,001 ; E S=1,0)$. A média de acertos da OT2 mostrou-se menor que OT3 $(Z=-6,042$; $p<$ $0,001 ; E S=0,87)$, de OT4 $(Z=-6,051 ; p<0,001 ; E S$ $=1,00)$ e de OT5 $(Z=-6,057 ; p<0,001 ; E S=1,00)$. Já OT3 apresentou-se menor que OT4 $(Z=-5,199 ; p$ $<0,001 ; E S=0,72)$ e de OT5 $(Z=-6,003 ; p<0,001$ $E S=0,95)$. E, a frequência de acerto de OT4 esteve menor que OT5 $(Z=-5,383 ; p<0,001 ; E S=0,74)$.

Outra variável analisada foi a confiança com a qual os participantes emitiram suas respostas de predição. A FIGURA 5 apresenta os valores da mediana e de intervalo entre o $1^{\circ}$ e o $3^{\circ}$ quartil, para a confiança dos grupos, em função das condiçôes de oclusão temporal.
Diferença significativa em comparação aos grupos 1GAD, 2GIM e 3GNO; e às condições aOT1, bOT2, cOT3, dOT4 e eOT5.

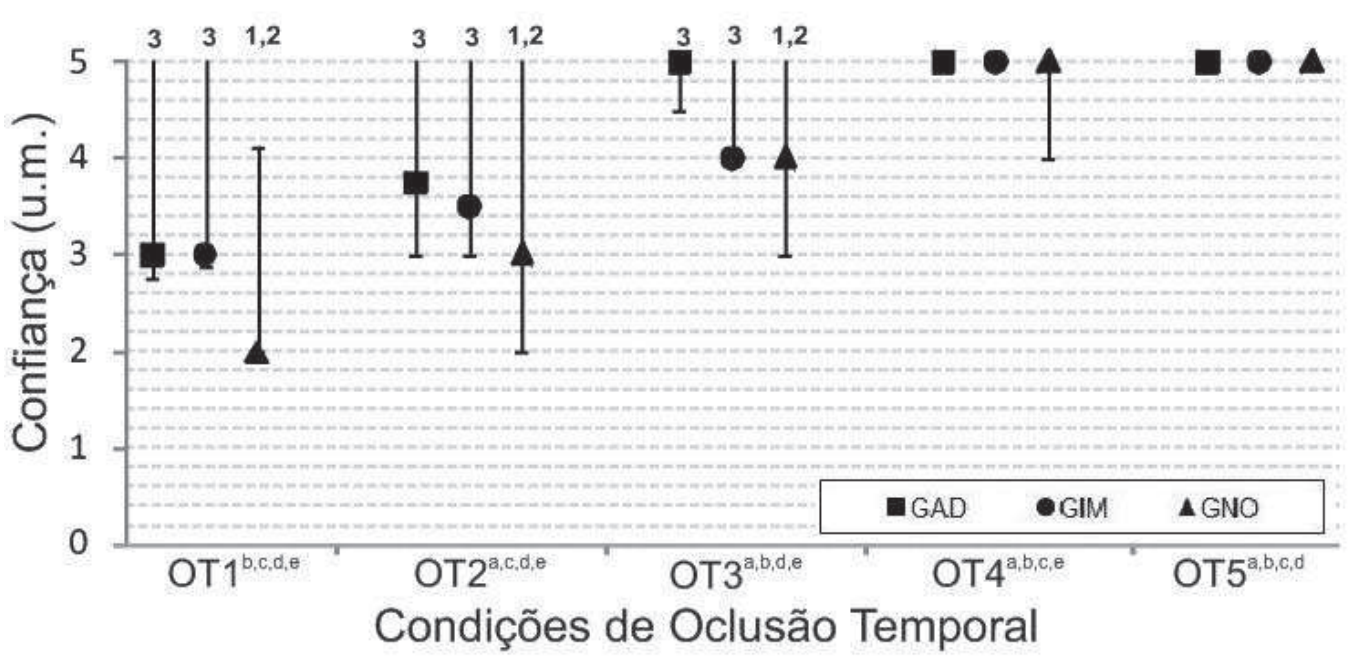

FIGURA 5 - Escores de confiança (mediana e intervalo entre o $1^{\circ}$ e o $3^{\circ}$ quartil) declarados pelos grupos para as respostas de predição, em função das condições de oclusão temporal analisadas.

$\mathrm{Na}$ análise inferencial foi encontrado efeito principal para o fator grupo nas condiçôes de oclusão OT1 $\left(H_{(2)}=13,975 ; p=0,001 ; E S=0,29\right)$, OT2 $\left(H_{(2)}=12,594 ; p=0,002 ; E S=0,26\right)$, OT3 $\left(H_{(2)}=\right.$ $19,901 ; p<0,001 ; E S=0,42)$ e OT4 $\left(H_{(2)}=6,701\right.$; $p=0,035 ; E S=0,14)$; e para o fator oclusão $\left(\chi^{2}{ }_{(4)}\right.$ $=165,507 ; p<0,001 ; E S=0,86)$.
$\mathrm{Na}$ análise do efeito para o fator grupo, a análise de "post hoc" foi novamente utilizada seguida da correçáo de Bonferroni e indicou a presença de diferenças estatísticas entre GAD e GNO em OT1 $(U=46,000 ; p=0,001 ; E S=0,64)$, OT2 $(U=$ $48,500 ; p=0,002 ; E S=0,62)$ е OT3 $(U=21,000$; $p<0,001 ; E S=0,83)$ e entre GIM e GNO também 
em OT1 $(U=48,500 ; p=0,002 ; E S=0,62)$, OT2 $(U=53,500 ; p=0,003 ; E S=0,58)$ e OT3 $(U=$ $54,500 ; p=0,003 ; E S=0,57)$ na qual, em ambas as comparaçôes, GAD e GIM se mostraram mais confiantes em suas respostas que GNO. O efeito encontrado entre os grupos para OT4 novamente não foi sensível após correçáo utilizada (Bonferroni's $p>0,016$ ).

$\mathrm{Na}$ análise do efeito para o fator oclusão, a confiança com a qual os indivíduos emitiram suas respostas diferiu entre si ao longo de todas as condiçôes (Bonferroni's $p<0,01$ ). Na condição OT1 $(\mathrm{Md}=3[2,3])$ os participantes apresentaram

\section{Discussão}

No presente estudo, foi analisado o efeito da oclusão temporal na cortada do voleibol sobre a tomada de decisão defensiva em atletas com diferentes níveis de experiência. Para tanto, o paradigma da oclusão temporal foi utilizado para interromper os vídeos em diferentes momentos a fim de identificar a capacidade de antecipação da identificação do local de aterrissagem da bola após uma jogada de ataque. A precisão e a confiança dos jogadores na realização desta antecipação foram analisadas em atletas de diferentes níveis de experiência.

$\mathrm{Na}$ literatura, poucos estudos realizaram comparaçóes entre categorias com base na faixa etária dos participantes, sendo que as análises têm sido normalmente realizadas considerando o período de experiência que um determinado grupo possui em uma modalidade ou tarefa. Sendo assim, o grupo de "novatos" será comparado aos "novatos" de outros estudos uma vez que este está presente em quase a totalidade dos estudos investigados. Por outro lado, para as comparaçóes com demais estudos da literatura, as categorias "adulto" e "infanto e mirim" desse estudo serão contrastadas com os grupos "experientes" e "intermediários", respectivamente.

A análise da frequência de acertos demonstrou superioridade dos grupos em relação à probabilidade de chance (25\%, ocasionado pelo delineamento empregado). Assim, independente da quantidade de experiência ou tipo de oclusão, os grupos demonstraram ser capazes de utilizar informaçóes visuais da ação, reconhecendo algum padrão, para realizar a predição da resposta. Esse resultado pode ter sido encontrado pelo fato da variável de análise ser apenas a precisão de resposta (acerto/erro), não sendo analisado o tempo menor confiança em relação a OT2 $(\mathrm{Md}=3[3,4])$ $(Z=-5,013 ; p>0,001 ; E S=0,48)$, OT3 $(\mathrm{Md}=$ $4[4,5])(Z=-5,907 ; p<0,001 ; E S=0,88)$, OT4 $(\mathrm{Md}=5[5,5])(Z=-5,974 ; p<0,001 ; E S=0,92)$ e OT5 $(\mathrm{Md}=5[5,5])(Z=-5,991 ; p<0,001 ; E S=$ $0,92)$. A confiança em OT2 foi menor que OT3 $(Z$ $=-5,360 ; p<0,001 ; E S=0,60)$, OT4 $(Z=-5,790 ; p$ $<0,001 ; E S=0,81)$ e OT5 $(Z=-5,856 ; p<0,001$; $E S=0,84)$. Na condição OT3 a confiança obteve menores valores que em OT4 $(Z=-4,859 ; p<0,001$; $E S=0,84)$ e OT5 $(Z=-5,068 ; p<0,001 ; E S=0,45)$. E, por fim, em OT4 a confiança apresentou-se menor que OT5 $(Z=-2,739 ; p<0,001 ; E S=0,04)$.

gasto pelos participantes para emitir suas respostas. Ainda que um tempo destinado à emissão das respostas pudesse estar previsto nos procedimentos, essa restrição não se equivale a de um atleta realizando uma ação de defesa em cenário real.

Os resultados, no que se refere aos grupos com maior experiência (GAD e GIM), corroboram os achados de Williams e Burwitz ${ }^{32}$, Shim et al. ${ }^{8}$ e JaCKson et al. ${ }^{13}$, cujos grupos experientes e intermediários apresentaram mais respostas corretas do que os menos experientes. Tais estudos avaliaram a antecipação de goleiros quanto a direçáo e a altura da bola em uma batida de pênalti, o tipo de batida realizada por um oponente no tênis e a mudança de direção realizada por atacantes no Rúgbi, respectivamente. Em contraposição, STARKes ${ }^{1}$ identificou que somente jogadores de hóquei experientes foram capazes de predizer a localização que a bola entrou no gol (seis áreas) em situaçóes anteriores ao contato entre taco e bola, com precisão superior à probabilidade de chance.

Vale destacar que a probabilidade de acerto ao acaso no estudo citado foi de $16,4 \%$ e no estudo atual foi de 25\%. Em CAÑAL-Bruland et al. ${ }^{2}$, avaliando a experiência perceptiva e percepto-motora de jogadores, técnicos e árbitros experientes e de novatos, encontraram uma superioridade frente à probabilidade de chance somente na condição de contato entre o atacante e a bola. Nas condiçóes que antecederam este contato (contato do levantador com a bola e no pico da bola após levantamento) os grupos, independente de sua experiência, não diferiram da probabilidade de chance.

Os resultados de prediçáo indicaram uma superioridade do grupo com maior experiência sobre os 
novatos apenas na condição em que o vídeo foi interrompido à $266 \mathrm{~ms}$ (oito quadros) antes do contato entre o atacante e a bola, e frente aos intermediários quando o vídeo foi interrompido à $133 \mathrm{~ms}$ (quatro quadros), também antes do contato entre o atacante e a bola. Estes resultados demonstram que a quantidade de experiência adquirida pelos participantes do grupo da categoria "adulta", ao longo de sua prática na modalidade, possibilitou-lhes um reconhecimento de padrão superior a de grupos com menor experiência, "infanto e mirim" e "novatos". Isso lhes permitiu predizer, numa frequência maior de acertos, o local de finalização dos ataques adversários, proporcionando, assim, um auxílio para açóes de defesa.

Conforme mencionado anteriormente, os correntes resultados corroboram o estudo de Williams e BURWITZ ${ }^{32}$ no fato dos goleiros com maior experiência no futebol terem apresentado capacidade de predição da altura da bola superior a dos novatos. Contudo, tais diferenças foram evidenciadas nas condiçóes anteriores ao contato do pé do batedor com a bola (120 ms e $40 \mathrm{~ms}$ ), o que vai de encontro aos achados do presente estudo, cujas diferenças deramse apenas em 266 ms anterior ao contato da máo do atacante com a bola. A partir de $133 \mathrm{~ms}$ anterior ao contato da mão do atacante com a bola os grupos com diferentes quantidades de experiência apresentaram resultados semelhantes.

Da mesma forma no badminton, ABERnethy ${ }^{4}$ encontrou uma superioridade de experientes frente aos novatos apenas à $83 \mathrm{~ms}$ antes do contato entre raquete e peteca, sendo que à $167 \mathrm{~ms}$ anterior ao contato não houve esta diferenciação entre os grupos. Para Cañal-Bruland et al. ${ }^{2}$, a experiência percepto-motora adquirida pelos atletas da modalidade de volei de praia ao longo de sua prática, proporciona aos mais experientes uma capacidade antecipatória superior aos novatos, contudo, esta diferença apenas se manifestou na condição de contato entre o atacante e a bola.

Além disso, outros estudos ${ }^{1,3,5,8,14,23,25,33}$ identificaram que a diferença apresentada entre os grupos se dá tanto em condiçôes anteriores quanto posteriores à 0 $\mathrm{ms}$ (no contato), demonstrando uma total superioridade dos experientes na utilizaçáo de informaçóes visuais para antecipação de um evento. Todavia, nos estudos conduzidos por CoelHo e CHAMBerLain ${ }^{15}$ e Williams et al. ${ }^{11}$ os autores verificaram que, ao contrário dos estudos acima citados, os grupos não se mostraram diferentes em suas prediçôes, com esta diferença apenas sendo identificada com relação ao tempo que levavam para emitir tais respostas.
Diferentemente da comparação entre experientes e novatos, poucos estudos incluíram em seu delineamento grupos com experiência intermediária. No presente estudo e, como citado anteriormente, os resultados demonstraram que a diferença entre grupos experientes e intermediários se dá apenas à 133 ms anterior ao contato. Estes resultados mostraram-se contrários a resultados anteriores, tais como nos estudos conduzidos por Starkes $^{1}$ e Tenenbaum et al. ${ }^{23}$, nos quais a superioridade dos experientes ocorreu tanto nas condiçôes pré quanto pós evento, sendo que, em Tenenbaum et al. ${ }^{23}$, também foi avaliado uma condição exatamente no momento do contato entre raquete e bolinha, a qual também apresentou a mesma superioridade. Estes estudos, diferentemente do presente, indicaram que o tempo intermediário de prática náo demonstrou ser suficiente para utilizar informaçóes visuais do movimento do oponente na mesma proporção que experientes.

$\mathrm{Na}$ comparação entre intermediários e novatos, não foi encontrada diferença significante na predição dos grupos em nenhuma das condiçóes, com o tempo de prática do grupo intermediário náo sendo suficiente para diferi-lo dos novatos em sua capacidade de antecipação. Estes resultados corroboram StARKes ${ }^{1}$ e IsAacs e Finch ${ }^{22}$, sendo que, estes últimos, analisaram apenas acerto e erro. Em Tenenbaum et al. ${ }^{23}$, os autores indicaram uma superioridade na predição de tenistas intermediários frente a novatos até $160 \mathrm{~ms}$ anterior ao contato entre a raquete e a bola, indicando que a maior quantidade de experiência possibilitaria uma melhor leitura do movimento do oponente.

Um aspecto a ser destacado foi a de que, independente da quantidade de experiência prévia, a quantidade de prediçóes corretas dos grupos aumentaram significantemente entre as condiçóes de oclusão, demonstrando assim que até $133 \mathrm{~ms}$ após o contato do atacante com a bola o defensor ainda tem à sua disposiçẫo informaçóes relevantes a serem utilizadas para identificação do local de aterrissagem da bola. Estes resultados corroboram os achados de Williams e Burwitz ${ }^{32}$ e Tenenbaum et al. ${ }^{23}$, e de certa forma, os resultados de ABERNETHY e RusseLL ${ }^{3}$, os quais identificaram uma reduçáo significante no número de erros até a condição de $83 \mathrm{~ms}$ após o contato. Contudo, os resultados parecem contrapor achados de STARKES et al. ${ }^{25}$, o qual identificou que a frequência de erros na prediçáo da localização de aterrissagem de uma bola sacada no voleibol diminui significantemente das condiçôes pré para o contato entre a mão do sacador e a bola, 
sendo que, após o contato as informaçóes se tornariam redundantes, náo apresentando melhora na capacidade antecipatória dos grupos.

Do mesmo modo, JACKSON et al. ${ }^{13}$ encontraram que, no rúgbi, o aumento significativo dos acertos da direção tomada por um oponente em uma situação 1 x 1 se deu até 120 ms anterior a troca de direção, com as informaçóes tornando-se redundantes a partir desse momento. Em CAÑAL-BrulAND et al. ${ }^{2}$ a predição de acertos somente apresentou ganho significativo da condição entre o pico de altura da bola após levantamento e o momento de contato entre o atacante e a bola, não sendo verificadas condiçóes pós-contato.

Vale destacar no presente estudo os achados referentes à utilização da análise da confiança com a qual os participantes emitiram suas respostas. Os resultados demonstraram que, tanto os participantes da categoria "adulta" quanto os da "infanto e mirim", apresentaram-se mais confiantes que os participantes novatos ao longo das condiçóes que vão até $133 \mathrm{~ms}$ anterior ao contato entre o atacante e a bola. Os dados demonstraram que a quantidade de experiência adquirida pelo atleta, ao longo de sua prática, aumenta sua confiança na antecipação de uma ação.

Esses resultados contrapóem-se aos achados de StARKES ${ }^{1}$ e JACKSON et al. ${ }^{13}$, os quais demonstraram que, jogadores de hóquei e de rúgbi com maior experiência não se diferenciam de participantes com menor ou nenhuma experiência, na confiança com que emitem uma resposta na tentativa de antecipar a localização que uma bola entra no gol e para indicar a direção de saída, tomada por um atacante, em uma situação $1 x 1$, respectivamente.

No estudo realizado por Tenenbaum et al. ${ }^{23}$, surpreendentemente, os autores encontraram uma inferioridade na confiança dos experientes em predizer o local de aterrissagem de uma bola após ser rebatida por um oponente nas condiçóes anteriores ao contato entre raquete e bola, demonstrando superioridade apenas nas condições pós. Estes resultados demonstraram que somente após $133 \mathrm{~ms}$ pré-contato, os participantes novatos são capazes de se igualarem a experientes e intermediários quanto a sua confiança na emissão das respostas corretas.

A ausência de diferenças na confiança com que grupos mais e menos experientes emitem suas respostas nos indica que, apesar de participantes com experiência intermediária apresentarem uma capacidade de antecipação inferior aos experientes, o tempo de engajamento na modalidade lhes permite apresentar confiança suficiente em suas respostas. Contrapondo estes achados Tenenbaum et al. ${ }^{23}$ não encontraram esta semelhança entre os jogadores de tênis experientes e intermediários, no qual experientes apresentaram uma maior confiança nas condições pós-contato. As contradiçóes apresentadas entre os estudos podem ter relação com as diferenças de exigências de cada uma das modalidades. Em se tratando somente da comparação de intermediários e novatos o presente estudo corrobora TENENBAuM et al. ${ }^{23}$ o qual justifica que a quantidade de prática adquirida pelos intermediários no decorrer de sua experiência com a modalidade já os possibilita apresentar uma confiança em suas respostas superior aos novatos nas condiçôes anteriores ao contato.

Os dados aqui obtidos dão suporte para algumas conclusóes. Primeiramente, as análises indicaram que, independente da experiência dos participantes, os grupos apresentaram-se acima da probabilidade de chance $(25 \%)$ no que diz respeito às prediçóes corretas, demonstrando assim, serem capazes de utilizar informaçóes do ambiente como auxílio na predição da localização de aterrissagem de uma bola, finalizada por um jogador adversário, em uma ação de defesa no voleibol. No entanto, o fato da avaliação se dar apenas quanto a predição (acerto/erro), não sendo avaliado o tempo cujos participantes levaram para emitir suas respostas, pode ter reduzido as possíveis possibilidades de diferenças estatísticas.

Em segundo lugar, apesar da capacidade antecipatória utilizada pelos grupos, a quantidade superior de experiência de GAD fez com que sua capacidade antecipatória se mostrasse superior a GNO à $266 \mathrm{~ms}$ e a GIM à 133 ms, ambas as condiçóes anteriores ao contato do atacante com a bola. Os dados mostraram que até 399 ms antes e após o contato da mão do atacante com a bola, a quantidade de experiência não discriminou os grupos quanto a sua frequência de prediçóes corretas. Assim, podemos inferir que até $399 \mathrm{~ms}$ antes do contato do atacante com a bola, apesar dos grupos apresentarem frequência de acertos superior ao nível de chance, as informaçôes pareceram não se mostrar tão relevantes. Ao contrário, nas condiçóes de contato ( 0 ms) e logo após o contato (133 ms) as informaçóes passaram a ser redundantes para os três grupos, não sendo identificada diferenças entre os mesmos. Importa-se saber também que a quantidade de experiência apresentada pelo grupo de intermediários, não foi capaz de discriminá-los dos novatos ao longo de todas as condiçôes.

No que se refere à confiança, concluiu-se que, se a quantidade de experiência apresentada por GIM não foi capaz de diferenciá-lo de GNO quanto à predição de respostas, a mesma foi capaz de auxiliá-los 
no aumento da confiança com a qual emitiam suas respostas, levando-os a assemelharem-se a GAD. Da mesma forma, foi visto que, se por um lado o GAD não se diferenciou dos demais quanto as suas predições nos vídeos interrompidos à $399 \mathrm{~ms}$ anterior ao contato do atacante com a bola, este se demonstrou mais confiante frente as suas respostas e que, semelhante aos dados de predição, a confiança dos grupos apresentou semelhanças a partir do contato do atacante com a bola.
De forma geral, podemos concluir que o tempo maior de prática (experiência) dos sujeitos, como citado nos estudos preliminares, auxilia os sujeitos no que se refere às habilidades cognitivas perceptuais, aqui analisadas por meio da tomada de decisão e antecipação na modalidade de Voleibol. De maneira alguma consideramos aqui sanadas todas as dúvidas referentes ao tema “Tomada de Decisão e Capacidade Antecipatória”. Os dados aqui encontrados são muito relevantes e deverão serem retomados e ampliados em estudos posteriores.

\title{
Notas
}

Todos os autores são membros do Grupo de Estudo e Pesquisa em Desenvolvimento e Aprendizagem Motora (GEPEDAM).

Os autores Fábio Luis Bordini, Marcelo Alves Costa e Thiago Viana Camata são alunos do Programa de Pós-graduação associado Universidade Estadual de Londrina e Universidade Estadual de Maringá (UEL/UEM) e as autoras Josiane Medina-Papst e Inara Marques são docentes da Universidade Estadual de Londrina.

\begin{abstract}
Effect of temporal occlusion of the attack action on defensive decision making in volleyball

This study examined the effect of temporal occlusion of a volleyball spike on the defensive decisionmaking of athletes with different levels of experience. The participants were divided in three groups: adult (GAD; $n=16)$, sub-17 (GIM; $n=16$ ) and novice adult (GNO; $n=16)$. Images of attacks performed by 4 athletes were edited in five different moments: (OT1) $399 \mathrm{~ms}$ (12 frames) before the hitter's contact with the ball, (0T2) $266 \mathrm{~ms}$ (eight frames) before contact; (0T3) $133 \mathrm{~ms}$ (four frames) before contact; (OT4) at the moment of hitter's contact with the ball and; (OT5) 133 ms (four frames) after the hitter's contact with the ball. After watching the edited videos, participants were asked to predict the landing site of the ball, followed by the confidence with which they issued their responses. The precision in the prediction of the ball's trajectory (correct/incorrect) and the confidence of the response (1-5 Likert scale) were measured. Regarding the correct response frequency, the $\operatorname{GAD}(\bar{X}=63.67 \pm 10.38 \%)$ group showed a greater frequency of correct responses than GIM $(\bar{X}=55.46 \pm 10.17 \%)$ in OT2 $(p=0.001)$ and the frequency of correct responses of GAD $(\bar{X}=79.29 \pm 10.38 \%)$ was also greater than GNO $(\bar{X}=$ $71.87 \pm 10.43 \%)$ in 0T3 $(p=0.012)$. The conditions were different from each other, with the frequency of correct responses increasing from OT1 $(\bar{X}=36.06 \pm 12.44 \%)$ to OT5 $(\bar{X}=98.17 \pm 4.81 \%)$. Regarding confidence, GAD and GIM were more confident than GNO in OT1, OT2 and OT3. Again, the conditions were different from each other, with the groups showing more confidence in OT5. We conclude that, regardless of experience, all groups were able to predict the landing site of the ball. However, groups with more experience were superior in their anticipatory ability.
\end{abstract}

KEY WoRDS: Decision-making; Anticipation; Visual recognition pattern; Volleyball. 


\section{Referências}

1. Starkes JL. Skill in field hockey: the nature of the cognitive advantage. J Sport Psychol. 1987;9:146-61.

2. Cañal-Bruland R, Mooren M, Savelsbergh GJP. Differentiating experts' anticipatory skills in beach volleyball. Res Q Exerc Sport. 2011;82:667-74.

3. Abernethy B, Russell DG. Expert-novice differences in an applied selective attention task. J Sport Psychol. 1987;9:326-46.

4. Abernethy B. Expert--novice differences in perception: how expert does the expert have to be? Can J Sport Sci. 1989;14:27-30.

5. Abernethy B. Anticipation in squash: differences in advance cue utilization between expert and novice players. J Sports Sci. 1990;8:17-34.

6. Tenenbaum G, Sar-El T, Bar-Eli M. Anticipation of ball location in low and high-skill performers: a developmental perspective. Psychol Sport Exerc. 2000;1:117-28.

7. Farrow D, Abernethy B, Jackson RC. Probing expert anticipation with the temporal occlusion paradigm: experimental investigations of some methodological issues. Motor Control. 2005;9:330-49.

8. Shim J, Carlton LG, Chow JW, Chae W-S. The use of anticipatory visual cues by highly skilled tennis players. J Motor Behav. 2005;37:164-75.

9. Shim J, Miller G, Lutz R. Visual cues and information used to anticipate tennis ball shot and placement. J Sport Behav. 2005;28:186-200.

10. Jackson RC, Mogan P. Advance visual information, awareness, and anticipation skill. J Motor Behav. 2007; 39:341-51.

11. Williams AM, Davids K, Burwitz L, Williams JG. Visual search estrategies in experienced and inexperienced soccer players. Res Q Exerc Sport. 1994;65:127-35.

12. Weissensteiner J, Abernethy B, Farrow D, Müller S. The development of anticipation: a cross-sectional examination of the practice experiences contributing to skill in cricket batting. J Sport Exerc Psychol. 2008;30:663-84.

13. Wackson RC, Warren S, Abernethy B. Anticipation skill and susceptibility to deceptive movement. Acta Psychol. 2006;123:355-71.

14. Wright DL, Pleasants F, Gomez-Meza M. Use of advanced visual cue sources in volleyball. J Sport Exerc Psychol. 1990;12:406-14.

15. Coelho AJ, Chamberlain CJ. Decision making in volleyball as a function of expertise. Meeting on North American Society for Psychology of Sport and Physical Activity; Asilmor, USA. Asilmor: North American Society for Psychology of Sport and Physical Activity; 1991.

16. Williams AM, Ward PA. Anticipation and decision making: exploring new horizons. In: Tenenbaum G, Eklund RC, editors. Handbook of sport psychology. 3rd ed. New Jersey: John Wiley; 2007. p.960.

17. Marteniuk RG. Information processing in motor skills. New York: Holt, Rinehart and Winston; 1976.

18. Schmidt RC, Wrisberg CA. Aprendizagem e performance motora: uma abordagem da aprendizagem baseada na situação. 4a ed. Porto Alegre: Artmed; 2010.

19. Poulton EC. On prediction in skilled movements. Psychol Bull. 1957;54:467-78.

20. Howarth C, Walsh WD, Abernethy B, Snyder C. A field examination of anticipation in squash. Aust J Sci Med Sport. 1984;16:6-10.

21. Mann DTY, Williams AM, Ward PA, Janelle CM. Perceptual-cognitive expertise in sport: a meta-analysis. J Sport Exerc Psychol. 2007;29:457-78.

22. Isaacs LD, Finch AE. Anticipatory timing of beginning and intermediate tennis players. Percept Mot Skills. 1983;57:451-4.

23. Tenenbaum G, Levy-Kolker N, Sade S, Liebermann DG, Lidor R. Anticipation and confidence of decisions related to skilled performance. Int J Sport Psychol. 1996;27:293-307.

24. Chamberlain CJ, Coelho AJ. The perceptual side of action: decision-making in sport. In: Starkes JL, Allard F, editors. Cognitive issues in motor expertise. 2nd ed. Netherlends: North-Holland; 1993. p.375.

25. Starkes JL, Edwards P, Dissanayake P, Dunn T. A new technology and field test of advance cue usage in volleyball. Res Q Exerc Sport. 1995;66:162-7.

26. Reilly T, Williams AM, Nevill AM, Franks A. A multidisciplinary approach to talent identification in soccer. J Sports Sci. 2000;18:695-702.

27. Williams AM, Reilly T. Talent identification and development in soccer. J Sports Sci. 2000;18:657-67.

28. Gaspar p, Ferreira JP, Pérez LMR. Tomadas de decisão no desporto: o seu ensino a jovens atletas. Rev Mackenzie Educ Fís Esporte. 2005;4:95-111. 
29. Confederaçâo Brasileira de Voleibol. Regras oficiais do voleibol. Rio de Janeiro: Sprint; 2009.

30. Bizzocchi CO. Voleibol de alto nível: da iniciação à competição. 2a ed. Barueri: Manole; 2004.

31. Bojikian JC, Bojikian LP. Ensinando voleibol. 4a ed. São Paulo: Phorte; 2008.

32. Williams AM, Burwitz L. Advance cue utilization in soccer. In: Reilly T, Clarys J, Stibbe A, editors. Second world congress of science and football. London: E\&EF Spon; 1993.

33. Abernethy B, Russell DG. The relationship between expertise and visual search strategy in a racquet sport. Hum Mov Sci. 1987;6:283-319.

\section{Agradecimentos}

Este trabalho teve apoio da CAPES e Fundação Araucária.

ENDEREÇO

Fábio Luis Bordini

Departamento de Educação Física Centro Universitário Filadélfia - UNIFIL

Av. Juscelino Kubitchek, 1626

Recebido para publicação: 13/07/2013

Revisado: 19/11/13

Aceito: 07/07/2014

86020-000 - Londrina - PR - BRASIL

e-mail: flbordini@gmail.com

118 • Rev Bras Educ Fís Esporte, (São Paulo) 2015 Jan-Mar; 29(1):107-18 